\title{
The King-Kong Effects: Improving Sensation of Walking in VR with Visual and Tactile Vibrations at each Step
}

\author{
Léo Terziman* \\ INSA / INRIA / DGA
}

\author{
Maud Marchal* \\ INSA / INRIA
}

\author{
Franck Multon* \\ Université Rennes 2 / INRIA
}

\author{
Bruno Arnaldi* \\ INSA / INRIA
}

Anatole Lécuyer*
INRIA

\begin{abstract}
In this paper we present novel sensory feedbacks named "KingKong Effects" to enhance the sensation of walking in virtual environments. King Kong Effects are inspired by special effects in movies in which the incoming of a gigantic creature is suggested by adding visual vibrations/pulses to the camera at each of its steps. In this paper, we propose to add artificial visual or tactile vibrations (King-Kong Effects or KKE) at each footstep detected (or simulated) during the virtual walk of the user. The user can be seated, and our system proposes to use vibrotactile tiles located under his/her feet for tactile rendering, in addition to the visual display. We have designed different kinds of KKE based on vertical or lateral oscillations, physical or metaphorical patterns, and one or two peaks for heal-toe contacts simulation. We have conducted different experiments to evaluate the preferences of users navigating with or without the various KKE. Taken together, our results identify the best choices for future uses of visual and tactile KKE, and they suggest a preference for multisensory combinations. Our King-Kong effects could be used in a variety of VR applications targeting the immersion of a user walking in a 3D virtual scene.
\end{abstract}

Index Terms: I.3.7 [Computer Graphics]: Three-Dimensional Graphics and Realism-Virtual Reality

\section{INTRODUCTION}

Immersion is fundamental in Virtual Reality (VR) where the user experiences and interacts with virtual worlds. In particular, navigation is one essential task in VR [2] for which immersion can be crucial. Many techniques have been developed to produce a strong sense of immersion in VR. However, immersion is not a notion exclusive to the VR. Indeed, breathtaking special effects have become common in Hollywood movies to make the experience of the spectators more intense and more realistic, creating a strong immersion feeling. For example, some movies use special visual effects to suggest the incoming of a gigantic creature. Visual vibrations/pulses are applied on the camera at each of the creature steps.

To improve immersion when navigating in Virtual Environments (VE), researchers have developed numerous kinds of sensory feedback. For example, Lécuyer et al. [9] proposed Camera Motions (CM) to reproduce the walking oscillations of the user's point of view during navigation in VR. The point of view oscillates in the VE to generate the visual flow that would be produced by a real walk. They demonstrated that their approach not only improves the user's sensation of walking in VE, but also his immersion [9].

Another solution to improve immersion in VR consists in cumulating different types of sensory feedback [2]. Visual feedback can be combined with audio or haptic feedback, for example, resulting in a fully multimodal walking simulation [19]. A typical example can be found in video games which provide not only visual feedback, but also auditory and sometimes vibrotactile modalities.

*e-mail: firstname.lastname@inria.fr

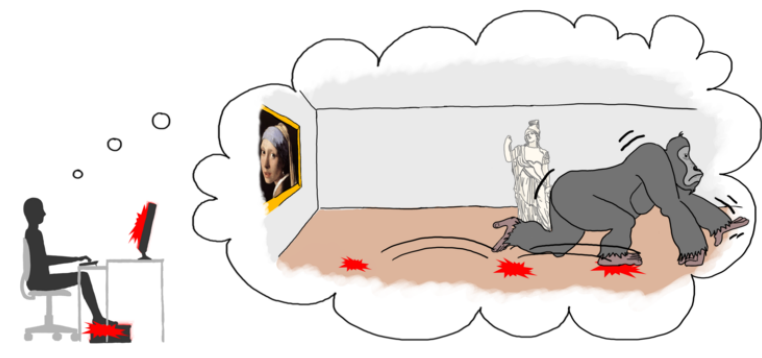

Figure 1: Concept of the King Kong Effects: Visual and Tactile vibrations inspired by special effects in movies enhance the sensation of walking in VE. Visual and Tactile feedbacks are generated at each step made in the VE.

In this paper, we propose a new technique to enhance the sensation of walking in VE inspired by special effects in Hollywood movies. The King Kong Effects (KKE) (Figure 1) provide a new kind of sensory feedback that simulates the feet touching the ground at each step by producing Visual and Tactile vibrations. The KKE can be used in a seated position, and we designed different vibration patterns based on physical and metaphorical models. As a result, we propose a new concept of sensory feedback effects, which correspond to the four main innovations claimed in our paper:

- A step simulator. We introduce a simple biomechanicallybased model to compute the footstep events, and the different contacts of the feet with the ground.

- New visual effects. We introduce the use of visual vibration patterns which simulate the contact of the feet with the ground at each step. Moreover, we simulate both the contacts of heel and toe. We propose and study vibration patterns along either vertical or horizontal directions.

- New vibrotactile effects. We also introduce new vibrotactile feedbacks generated under the feet of the user to reproduce the step sensation. Again, we simulate both the contacts of the heel and toe. Moreover, we propose two different vibration pattern metaphors: (1) a physically based metaphor and (2) a metaphor where the stimulation is proportional to the force pressure applied by the feet on the ground.

- Evaluation. Finally, we evaluated the different vibration patterns for both modalities. We also investigated the influence on the Visual KKE of another visual technique, the Camera Motions [9]. We also evaluated the KKE in a multimodal context with the modalities taken individually or all together.

The remainder of this paper is organized as follows: Section 2 provides an overview of the existing sensory feedback techniques for enhancing walking in VE. In Section 3 we introduce the King Kong Effects (KKE). We detail the Step Simulator used to generate the footstep events and we introduce our novel visual and vibrotactile patterns. In Section 4 we describe the results of a set of experiments conducted to identify the best parameters and best combinations of KKE. 


\section{Related Work: Improving Sensation of Walking IN VE}

Navigation is one of the essential tasks necessary to interact in VR [2]. To improve the range of sensory feedback available, many techniques require the users to physically walk in the real world. However, the working space is usually smaller than the VE, thus the users can not walk freely. The first solution uses treadmills [5] to keep the user at the center of the workspace. Other possibilities include mechanically actuated platforms under the user's feet [7] or moving tiles [6] to keep the user in place. However, these locomotion interfaces provide only kinaesthetic information to the user.

Other devices can be used to improve the range of simulated modalities (audio, visual, tactile). For example, the ALive Floor [16] is composed of actuated tiles that can simulate uneven grounds. Vibrotactile information can also be provided through shoe-based devices [14]. Using contact sensors and vibrators, the shoes can simulate different ground types by producing adapted vibrotactile feedback at each step. Another possibility uses tiled floors where each tile possesses force sensors and a vibrotactile actuator [18]. With this device, the user walks on a tiled floor and the foot pressure is used to track its position.

Audio feedback can also be adapted to simulate the different types of ground in the VE. For example, Serafin et al. [15] proposed a technique to extract the components of a real footstep sound in real time. This technique allows them to dynamically generate a new footstep sound, in real time, matching the properties of the virtual ground to be simulated. For example, a user walking on concrete in the real world could hear sounds of footsteps on snow. The physical simulation used allows a large variety of simulation of footstep sounds matching many types of virtual grounds such as snow, water, leafs, wood, concrete, gravel and so on [11].

Different modalities can also be combined to improve immersion when walking in VE. For example, shoe-based devices can be associated with real time audio simulation [14]. Contact sensors embedded in shoes are used to detect footsteps and both vibrotactile and auditory feedback are provided to match a specific virtual ground surface. Tactile tiles can also be used with spatialized audio in a CAVE to provide a complete simulation using the haptic, auditory and visual modalities [19].

However, some applications should be able to run in desktop mode, i.e. when the user is seated and is using a basic computer. This includes training applications that need to be massively deployed, or video games. To give the sensation of walking, video games use auditory feedback intensively and footstep sounds to simulate steps. Visual information can also be used to enhance the sensation of walk in VE. For example, effects on the virtual camera can be created [9]. Camera Motions (CM) simulate the motions of the user's head and its associated visual flow during the walk [9]. The point of view of the user oscillates to follow the head motions that would be produced by a real walk. Compensation of the head orientation to focus the gaze on the objects is known as the oculomotor reflex. This reflex can also be added to CM [4], which are then adapted to follow the user's gaze in real time [3]. Moreover, $\mathrm{CM}$ can also be used to suggest slopes for example [10]. The height, advance speed and orientation of the camera can be used separately or together to inform about the slope. When the three parameters are combined the perception of slopes increase [10].

Unfortunately, the range of available modalities for sensory feedback in desktop mode is still very limited. In particular, to our best knowledge, vibrotactile feedback has been used scarcely in such context, and no visual feedback has been specifically developed to stress the footsteps during the walk. In the remainder of this paper, we present the King Kong Effects (KKE), a new set of sensory feedbacks designed to improve the sensation of walking by focusing on the perception of the footsteps during the walk through visual and tactile modalities.

\section{Concept of King Kong Effects}

We propose a new technique to enhance the sensation of walking in VE in desktop mode. The KKE is based on visual and tactile vibration patterns generated at each virtual step to simulate the contacts of the feet with the ground. Our technique can be used in static position, such as when seated or standing, whereas the user controls the virtual walk with any input device (joystick, keyboard, etc.).

The KKE are inspired from famous Hollywood movies such as King Kong or Godzilla where the walk of gigantic creatures is emphasized to make the spectators "feel" the steps of the incoming creature. For instance, Jurassic Park's T-Rex produces earth vibrations which generate waves in water, while Godzilla generates electrical disturbances. More recently, complex motions of the camera were used in movies like King Kong or Transformers to achieve the same goal. Our effects are, in a way, reproducing the special effects demonstrated in these movies for desktop VR technologies for the user himself, and are thus named "King Kong Effects" (KKE).

At each virtual step, the user can feel the sensation of hurting the ground with both visual vibration of the camera and vibrotactile feedback under his feet. For each modality, we propose a set of different vibration patterns. Visual and tactile vibrations can be used individually or together for a multimodal simulation. Moreover, existing auditory simulation of footstep sounds could be easily added to the KKE for even higher immersion.

The software architecture behind KKE is composed of three parts: the Step Simulator (1) is designed to compute footstep events based on a simple biomechanical model. Then, two different sensory feedback components corresponding to the visual (2) and vibrotactile (3) modalities have been developed to enhance the walk in the VE based on the generated footstep events (Figure 2).

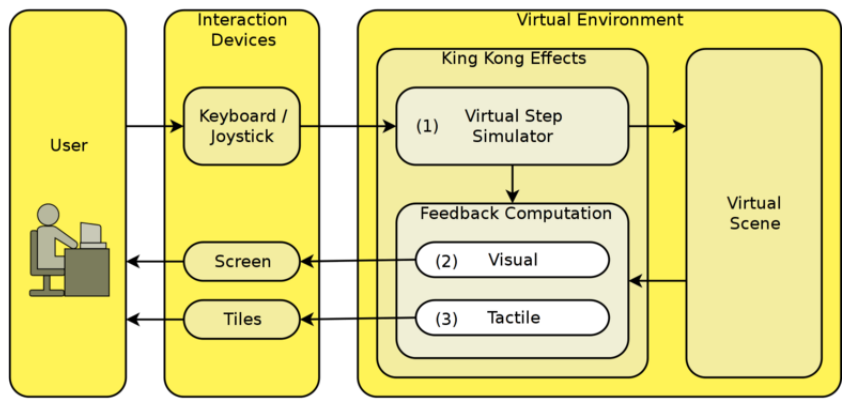

Figure 2: Architecture of the KKE. The user inputs are processed by the Step Simulator to generate footstep events. The events are used to generate visual and vibrotactile feedback.

\subsection{Step Simulator}

To compute the virtual footsteps events in the VE, we need a simple yet realistic biomechanical model. Indeed, contrarily to existing techniques, the KKE do not rely on force sensors to detect the footsteps. Indeed, the users should have the possibility to use the KKE even when seated at a desk using desktop VR applications, in which case the force sensors would be ineffective. However, they must be generated in a realistic way accurately reflecting the different user's interactions. Furthermore, the system must be compatible with different kinds of interaction devices, like a keyboard or a joystick for example.

To solve this problem, we propose a biomechanically inspired model that can generate footstep events in real-time based on a given advance speed. First, we use the classical decomposition of the walking motion in events given by Vaughan [17]: (1) Heel Strike (HS), (2) Heel Off (HO), (3) Toe Strike (TS) and (4) Toe Off (TO). Using this formalism, most of the human walking gaits can 


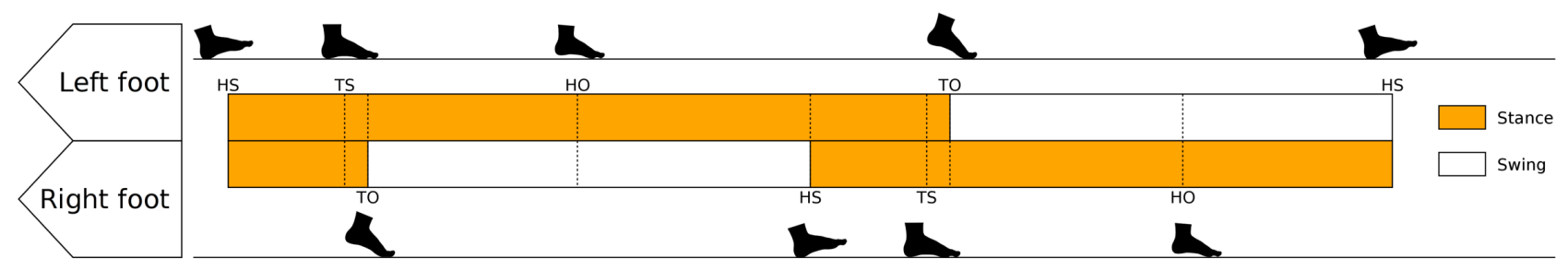

Figure 3: Walking cycle. The events of contact and separation of the heels and toes are placed accordingly to the position of the feet during the cycle. The parts of the cycle in orange correspond to the moments while either foot is in contact with the ground.

be described as a succession of different events in a precise time and order. For example, walking, running, sprinting or even jumping can be described by such sequences associated with a global advance speed. The positions of these events during the walking cycle are shown in Figure 3.

The position of each event in the gait cycle can be predicted. In our implementation, we used the data presented by Novacheck [12] for a regular walking motion, ie if the half-cycle is defined to start with $\mathrm{HS}$, TS and $\mathrm{HO}$ will be at $21 \%$ and $60 \%$ of the half-cycle respectively, while TO and HS of the opposite foot will happen at $24 \%$ and $100 \%$ respectively (Figure 3 ).

To generate the events, we need to determine the gait half-cycle length $T$, to know the accomplished percentage of the cycle. For the walk, the advance speed is given by [1]:

$$
v=L * f
$$

where $v$ is the advance speed, $L$ the step length and $f$ the step frequency defined as $\frac{1}{T}$. Thus we have:

$$
T=\frac{L}{v}
$$

However, while $v$ is obviously dependent on the users interactions, it can be easier to compute it from a factor of the interactions and the speed of comfort $v_{c}$ for a given gait. For the walk, the speed of comfort can be estimated using a simple model interpolated from experimental data [8]:

$$
L \sim v_{c}^{\beta}
$$

with $\beta$ being a constant which equals to 0.42 for adults. With $I$ the input speed in percentage of the comfort speed, such as $v=I * v_{c}$, we obtain:

$$
T=\frac{L}{I * e^{\frac{\ln (L)}{\beta}}}
$$

Thus, $T$ depends on $I$ : the gait cycle length can change in the middle of one step, which would be unrealistic and could induce implementation troubles. Thus, the value of $T$ must be updated only at the beginning of each new step.

For example, for a step length $L=1 \mathrm{~m}$, at the comfort speed, the duration of one step $T$ is 1 second, and thus the contact of the toe will happen 210 milliseconds after the contact of the heel of the same foot. Similarly, the heel will be off the ground 600 milliseconds after its initial contact, and the toe of the opposite foot will leave the ground 240 milliseconds after the initial heel strike.

Finally, our Step Simulator can generate footstep events using only a percentage of the comfort advance speed and the virtual avatar size as input. The events are generated using a simple biomechanical model, and can be used to synchronize our KKE.

\subsection{Visual King Kong Effects}

Based on the generated events for the heels and toes contact, each step can be visually simulated using KKE: the technique is based on the metaphor of a visual vibrations produced by each step.
A first metaphor considers the vibration from the point of view of the "creature" as a result of the feet hurting the ground and thus producing Vertical (V) vibrations. Moreover, a second metaphor emphasis the point of view from the environment point of view, as each step produce Horizontal (H) seismic vibrations when the feet of a heavy virtual avatar hit the ground.

We simulated two types of vibration patterns with 1 and 2 successive contacts of the feet with the ground respectively: contact of the heel alone ( 1 contact) as used in most movies, and a combination of the heel and toe strike together ( 2 contacts) to provide a more realistic biomechanical simulation.

All different combinations were tested resulting in 4 different types of vibrations (Figure 4). Thus, the Visual KKE can be described by the function $K_{V}(x, y)$ with $x$ the direction of the vibrations $(x \in\{V, H\})$ and $y$ the number of contacts $(y \in\{1,2\})$.

The vibration model is based on a Rigid Contact Model (RCM) used in haptic simulations for the contact between two rigid objects [13]. The vibrations of the camera are based on high frequency sinusoidal oscillations with an exponentially decaying envelope:

$$
Q(t)=A(v) e^{-B t} \sin (\omega t)
$$

where $Q(t)$ is the produced vibration, $A(v)$ the attack depending of the starting velocity $v, \omega$ the frequency of the oscillations and for a given material $B$ is the decay constant of the envelope.
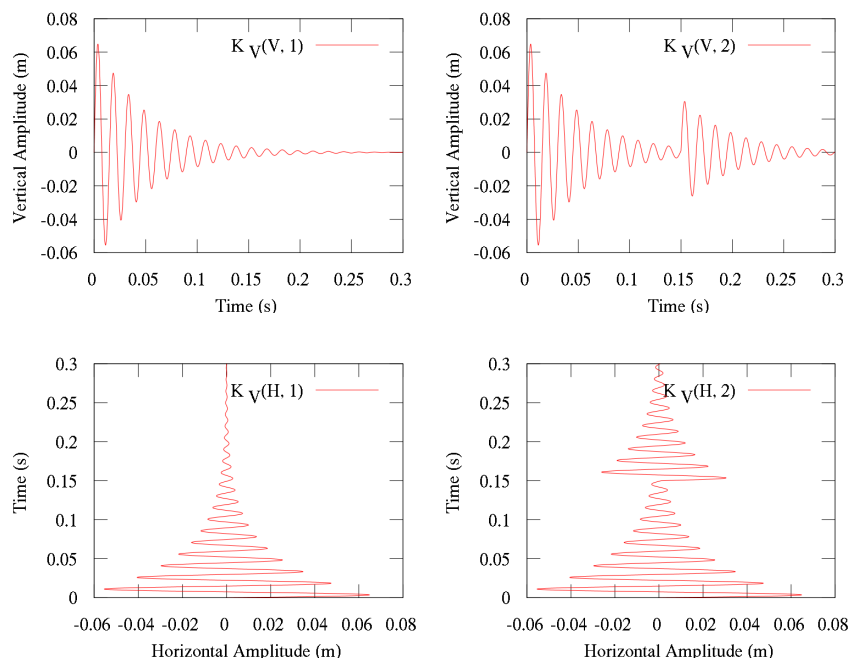

Figure 4: Vibration patterns for the Visual KKE. Amplitude (in meters) over Time (in seconds) of the vibration patterns used for the Visual KKE: 1 or 2 contacts in Vertical and Horizontal directions.

Values of $\omega$ and $B$ can be found for different materials in the literature [13]. However, the visual rendering strongly depends on 
the display device frame rate. If the frame rate is too low or the vibration frequency too fast, the user will not be able to perceive the vibration correctly. After preliminary testing, the decaying value $B$ was set to be twice the interval between HS and TS, ie $0.3 s$ and we used a constant amplitude for the attack $\left(A_{H S}=7 \mathrm{~cm}\right.$ and $A_{T S}=3 \mathrm{~cm}$ ). Finally, our preliminary tests showed that the value of $\omega$ should be inferior to twice the display frequency. In our implementation, we chose $\omega=67 \mathrm{~Hz}$ corresponding to a ground made of wood.

\subsection{Tactile King Kong Effects}

We also designed a set of vibrotactile techniques to stimulate the feet of the users. The vibrations were transmitted using low frequency loud speakers fixed on tiles under the users' feet [18] (Figure 5).

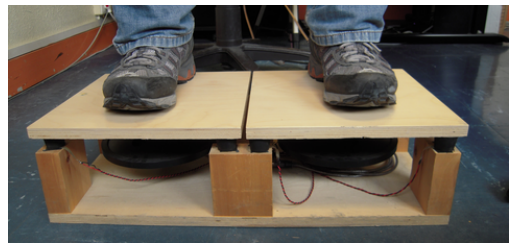

Figure 5: Vibrotactile tiles. The vibrations are produced by low frequency loud speakers fixed under the tiles [18].

Again, we simulated two types of contact of the feet with the ground: Heel Strike (1 contact) and both Heel Strike and Toe Strike ( 2 contacts).

We also developed 2 different metaphors for the vibrations. The first metaphor is a physically-based simulation of the vibrations that would be produced in real life using a Rigid Contact Model (RCM) [13] presented section 3.2. With this model, the user's feet are considered to be a rigid object colliding with a rigid surface. The resulting collision produces high frequency vibrations depending of the nature of the virtual ground. In the second metaphor, the vibrations are proportional to the forces applied on the ground by the feet: the Ground Reaction Forces Model (GRFM) simulates the force that is applied to the ground by each step [12]. We used the same vibration frequency as with the RCM model, but different envelopes are used for the signal. The envelopes reproduce the shape of the forces applied to the ground during each step [12]. For the heel contact only, only the beginning of the curve is interpolated from the data, while all the data is used otherwise. Thus, the Tactile KKE can be described by the function $K_{T}(x, y)$ with $x$ the model used $(x \in\{R C M, G R F M\})$ and $y$ the number of contacts $(y \in\{1,2\})$. The four resulting different vibration patterns are presented in Figure 6.

\subsection{Conclusion}

To sum up, our approach is composed of (1) a Step Simulator which computes the footstep events during the walk, (2) Visual vibration patterns based on vibrations along two different directions (vertical and horizontal) to produce different effects and (3) Tactile vibration patterns based on two different metaphors. Moreover, the different patterns can simulate both the heel and toe contacts with the ground. The KKE can be used in static position, such as when seated or standing, whereas the user controls the virtual walk with any input device (joystick, keyboard, etc.). Finally, the KKE can be implemented on any kind of computer, requiring only the tiles which are a low cost device.

\section{Evaluations}

We conducted a set of experiments to evaluate the different components of the KKE and to determine the best combination among
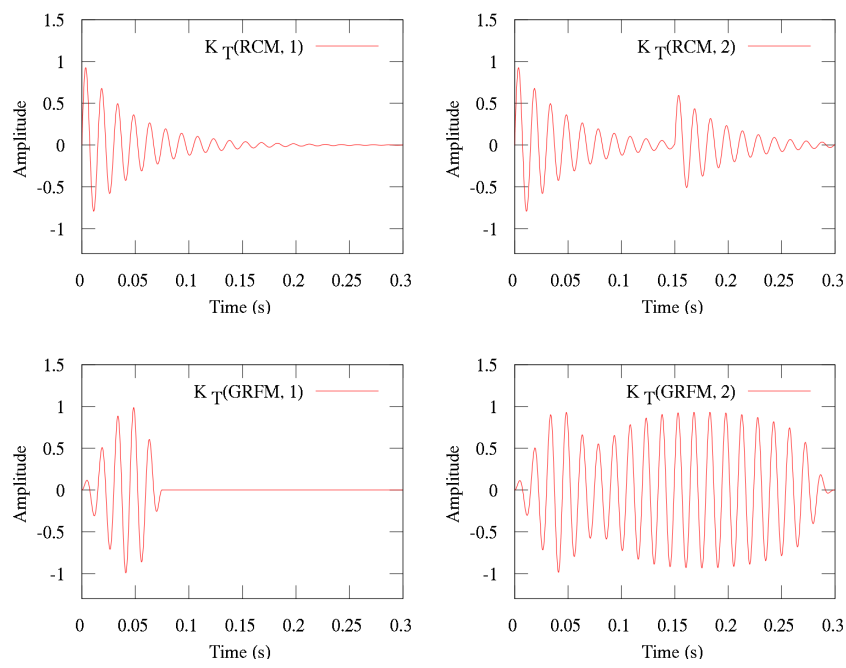

Figure 6: Vibration patterns for the tactile KKE. Amplitude over Time (in seconds) of the vibration patterns used for the Tactile KKE: Rigid Contact Model (RCM) and Ground Reaction Forces Model (GRFM) simulating 1 or 2 contacts.

modalities and effects.

We conducted four different experiments. The first three experiments were based on the 2 Alternative Forced Choice (2AFC) paradigm. For each experiment, the participants were exposed to pairs of passive navigations in straight line using different conditions. At the end of each pair, the participants were asked which one of the two navigations gave them the best sensation of walking. In every case, the participants had to choose between the two navigations. The first experiment was designed to evaluate the optimal parameters for the Visual KKE. The second investigated the influence of Camera Motions on the appreciation of Visual KKE. The third experiment focused on the optimal parameters for the tactile KKE. Finally, the fourth experiment tested the preference for the $\mathrm{KKE}$ in a multimodal context.

\subsection{Method}

\subsubsection{Experimental Apparatus}

The participants were seated at $1 \mathrm{~m}$ in front of a 24 inch widescreen monitor with a resolution of $1920 \times 1200$ pixels (physical field of view of $29^{\circ}$ horizontally and $18^{\circ}$ vertically). The rendering was made at a refresh rate of $50 \mathrm{~Hz}$. Their feet were placed on top of the vibrating tiles, with their shoes removed (Figure 7a). Users were wearing headphones filled with white noise to mask any sound produced by the vibrating tiles. At the end of each pair of navigations, the users had to select their preferred navigation in terms of "sensation of walking" using the keyboard keys " 1 " and " 2 ". The participants had the possibility to take breaks by pressing the "Space" key at any time.

\subsubsection{Virtual Environment}

The Virtual Environment was composed of an empty room with textured walls (Figure $7 \mathrm{~b}$ ). The room depth was set to $15 \mathrm{~m}$. At the end of the room, in the center of the screen, a cardboard box regularly textured was placed to provide the participants with a point to focus their gaze (Figure 7b). The participants were exposed to pairs of passive navigations of $5.4 \mathrm{~m}$. 


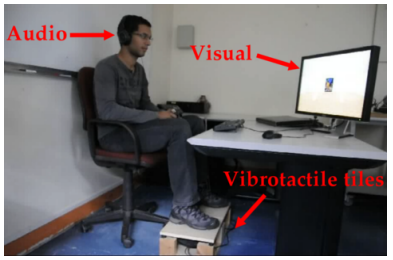

(a) Setup

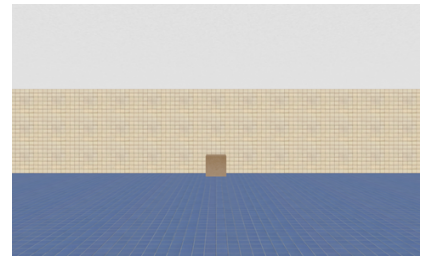

(b) Virtual Scene
Figure 7: Experimental apparatus.

\subsubsection{Collected Data}

For each pair of conditions, we recorded the choices of the participants. At the end of the experiment the participants had to complete a questionnaire in which they had to grade from 1 (very bad) to 7 (very good) the different techniques according to different criteria: (1) Presence, (2) Sensation of walking, (3) Realism of the walk, (4) Visual tiredness (5) Cybersickness and (6) Global appreciation. For the Visual tiredness and Cybersickness conditions, the grade 1 corresponded to "very tiresome" and "make sick" respectively, while the grade 7 was the opposite.

\subsection{Experiment 1: Selecting the Best Visual Vibration Pattern for KKE}

The first experiment was focused on Visual KKE. The goal was to find which set of parameters provides the best sensation of walking in the VE when the Visual KKE are used alone. The parameters were the number of contacts ( 1 contact versus 2 contacts) and the direction of oscillations (Vertical versus Horizontal).

\subsubsection{Population}

Twelve participants (10 males and 2 females) aged from 20 to 34 (mean $=23.8$, standard deviation $=3.5$ ) performed the experiment. None of the participants had any known perception disorder. All participants were used to VEs but were naïve with respect to the proposed techniques, as well as to the experimental setup.

\subsubsection{Experimental Conditions}

We used a within subject design to evaluate five different Visual KKE conditions. The control condition $\mathrm{Ctrl}$ was composed of a linear camera motion without any Visual KKE. The four other conditions corresponded to: (1) $K_{V}(V, 1)$, (2) $K_{V}(V, 2)$, (3) $K_{V}(H, 1)$ and (4) $K_{V}(H, 2)$. All the possible combinations of the different conditions were tested 10 times in both orders. For each group of possible combinations, the order between the different pairs was randomized. The experiment lasted approximatively 25 minutes.

\subsubsection{Results}

We analyzed answers and preferences of participants for the different patterns in order to determine which condition provides the best sensation of walking in the VE. In particular, we analyzed the impact of the direction of the oscillations, as well as the effect of one contact versus two contacts.

For a given pair of conditions, each individual performed 20 comparisons. Under the null hypothesis of equal preference between the two conditions, the number of times an individual preferred the first condition follows a binomial distribution with parameters 10 and 1/2. After standardization, such variable can be approximated by a standard normal random variable. Thus, for each pair of conditions, we tested the presence of a preferred condition using a Student's t-test. The p-values were adjusted with a Bonferroni correction. The analysis showed that $K_{V}(V, 1)$ was more often significantly chosen than $C t r l(t(11)=6.14, p<0.001), K_{V}(H, 1)$ $(t(11)=11.49, p<0.001)$ and $K_{V}(H, 2)(t(11)=7.58, p<0.001)$. Moreover, $K_{V}(V, 2)$ was more often significantly chosen than $C t r l$ $(t(11)=5.50, p=0.002), K_{V}(H, 1)(t(11)=9.57, p<0.001)$ and $K_{V}(H, 2)(t(11)=5.45, p=0.002)$. Ours results suggest that for the Visual KKE, the Vertical vibrations are always preferred over the Horizontal ones. Moreover, the number of contacts with the ground does not change this result. However, the experiment failed to found any significant effect on the number of contacts of the feet ( 1 or 2 contacts) for the Visual vibrations.

Concerning the subjective questionnaires, we performed a Friedman test. The reported p-values were adjusted for multiple comparisons. We found a significant effect for 5 criteria: Global appreciation $\left(\chi^{2}=3.6, p=0.003\right)$, Presence $\left(\chi^{2}=3.03, p=0.02\right)$, Realism $\left(\chi^{2}=4.27, p<0.001\right)$, Walking sensation $\left(\chi^{2}=3.96\right.$, $p<0.001)$ and Fatigue $\left(\chi^{2}=4.91, p<0.001\right)$. Post-hoc analysis showed that $K_{V}(V, 1)$ was preferred to $C t r l$ for global appreciation $(p=0.01)$, presence $(p=0.02)$, realism $(p<0.001)$ and walking sensation $(p<0.001) . K_{V}(V, 1)$ was also significantly better rated than $K_{V}(H, 1)$ for global appreciation $(p=0.003)$ and realism $(p=0.04)$, and significantly better rated than $K_{V}(H, 2)$ for global appreciation $(p=0.006)$, walking sensation $(p=0.02)$ and fatigue $(p=0.03)$. Moreover, $K_{V}(V, 2)$ was preferred to $C t r l$ for realism $(p=0.002)$, walking sensation $(p=0.046)$ and fatigue $(p=0.006)$. Finally, $K_{V}(H, 1)$ and $K_{V}(H, 2)$ were significantly better rated than Ctrl for fatigue only $(p<0.001$ and $p<0.001)$. The results of the questionnaires are displayed in Figure 8.

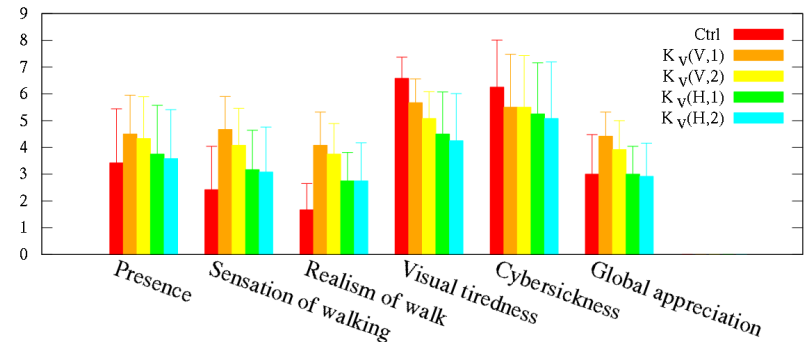

Figure 8: Results of the questionnaires for the first experiment. For each of the criteria, the mean and standard deviation for each condition are represented.

The results of the subjective questionnaires confirm the results of the 2AFC. Indeed, the Vertical vibrations with 1 contact scored better than the other conditions with Horizontal vibrations in terms of global appreciation and either realism or walking sensation.

\subsubsection{Conclusion}

To sum up, for the Visual KKE the Vertical direction provides a better sensation of walking than the Horizontal direction. In particular, the Vertical Visual KKE with 1 contact was preferred by the participants over the other conditions.

\subsection{Experiment 2: Testing the Combination of Visual KKE and Oscillating CM}

The second experiment tested the influence of standard oscillating Camera Motions on Visual KKE. The goal was to check if the preference for Visual KKE (number of contacts, direction of oscillations) would change when associated with another visual technique composed of standard camera motions.

\subsubsection{Population}

The same twelve participants as the first experiment performed this experiment. However, to avoid any bias, half of the participants started with this experiment while the other half started with the first one. 


\subsubsection{Experimental Conditions}

We used a within subject design to evaluate five different Visual KKE when added to the regular Camera Motions proposed by Lécuyer et al. [9]. The control condition $C t r l$ was made of classical camera motions composed of sinusoidal oscillations along the three axes, without any Visual KKE. The conditions were all composed of the camera motions combined respectively with: (1) $K_{V}(V, 1)$, (2) $K_{V}(V, 2)$, (3) $K_{V}(H, 1)$ and (4) $K_{V}(H, 2)$. All the possible combinations of the different conditions were tested 10 times in both orders. For each group of possible combinations, the order between the different pairs was randomized. The experiment lasted approximatively 25 minutes.

\subsubsection{Results}

We performed the same statistical analysis as for Experiment 1 . The analysis showed that $K_{V}(V, 1)$ was more often significantly chosen than $K_{V}(H, 1)(t(11)=4.99, p=0.004)$. Moreover, $K_{V}(V, 2)$ was more often significantly chosen than $K_{V}(H, 2)(t(11)=4.17, p=$ 0.016). Ours analysis suggest similar results to the first experiment: the Vertical vibrations are still preferred to the Horizontal ones. The presence and combination with Camera Motions do not change this result. Using the KKE in combination with standard Camera Motions does not modify the way users perceive the KKE, and the most efficient type of KKE remains the same. The analysis also failed to found any significant effect of the number of contacts of the feet (1 or 2 contacts) when combined with Camera Motions.

Concerning the subjective questionnaires, we performed a Friedman test. The reported p-values were adjusted for multiple comparisons. We found a significant effect for 5 criteria: Global appreciation $\left(\chi^{2}=4.52, p<0.001\right)$, Presence $\left(\chi^{2}=3.14, p=0.01\right)$, Realism $\left(\chi^{2}=4.38, p<0.001\right)$, Walking sensation $\left(\chi^{2}=4.89\right.$, $p<0.001)$ and Fatigue $\left(\chi^{2}=3.23, p=0.01\right)$. Post-hoc analysis showed that $K_{V}(V, 1)$ was preferred to $C t r l$ for presence $(p=0.04)$. $K_{V}(V, 1)$ was also significantly better rated than $K_{V}(H, 2)$ for presence $(p=0.02)$ and fatigue $(p=0.01)$. Moreover, $K_{V}(V, 2)$ was significantly better rated than $K_{V}(V, 1)$ for global appreciation $(p<$ $0.001)$, realism $(p<0.001)$ and walking sensation $(p<0.001)$. $K_{V}(V, 2)$ was also preferred to $K_{V}(H, 2)$ for global appreciation $(p<0.001)$ and walking sensation $(p=0.01)$. Finally, $K_{V}(H, 1)$ was significantly better rated than $K_{V}(V, 1)$ for global appreciation $(p=0.008)$, realism $(p=0.01)$ and walking sensation $(p=0.02)$. The results of the questionnaires are displayed in Figure 9.

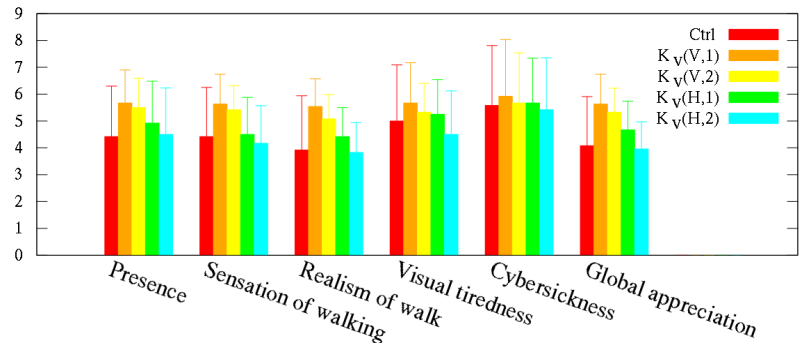

Figure 9: Results of the questionnaires for the second experiment. For each of the criteria, the mean and standard deviation for each condition are represented.

The results of the questionnaires are more contrasted. Indeed, Vertical vibrations scored better than Horizontal vibrations for walking sensation and global appreciation with 2 contacts, while it is the opposite for 1 contact.

\subsubsection{Conclusion}

To sum up, combined with CM, the Vertical direction of the Visual KKE is still preferred over the Horizontal direction.

\subsection{Experiment 3: Selecting the Best Tactile Vibration Pattern for KKE}

The third experiment focused on tactile KKE. The goal was to find which set of parameters provides the best sensation of walking in the VE when the tactile KKE are used alone. The parameters were the number of contacts ( 1 contact versus 2 contacts) and the model used (RCM versus GRFM).

\subsubsection{Population}

Twelve participants different from the participants from the two previous experiments ( 8 males and 4 females) aged from 21 to 59 (mean $=30.1$, standard deviation $=12.6$ ) performed the experiment. None of the participants had any known perception disorder. All participants were used to VEs but were naïve with respect to the proposed techniques, as well as to the experimental setup.

\subsubsection{Experimental Conditions}

We used a within subject design to evaluate five different Tactile KKE conditions. The control condition $C t r l$ was composed of no Tactile KKE. The conditions were respectively: (1) $K_{T}(R C M, 1)$, (2) $K_{T}(R C M, 2)$, (3) $K_{T}(G R F M, 1)$ and (4) $K_{T}(G R F M, 2)$. All the possible combinations of the different conditions were tested 10 times in both orders. For each group of possible combinations, the order between the different pairs was randomized. The experiment lasted approximatively 25 minutes.

\subsubsection{Results}

We performed the same statistical analysis as in Experiment 1. The analysis showed that $K_{T}(R C M, 1)$ was more often significantly chosen than $\operatorname{Ctrl}(t(11)=36.38, p<0.001), K_{T}(R C M, 2)(t(11)=$ $4.26, p=0.0133)$ and $K_{T}(G R F M, 2)(t(11)=4.64, p=0.0072)$. Moreover, $K_{T}(G R F M, 1)$ was more often significantly chosen than $K_{T}(R C M, 2)(t(11)=5.46, p=0.002)$ and $K_{T}(G R F M, 2)(t(11)=$ $8.25, p<0.001)$. Concerning the vibration patterns, no significant effect on the metaphor was found during the 2AFC analysis. Rigid Contact Model (RCM) with only heel strikes was preferred to the Ground Reaction Forces Model (GRFM) with heel and toe strikes. On the other hand, the GRFM with only heel strikes was also found significantly preferred to the RCM with heel and toe strikes. Thus, the number of contacts seems to be a more important criterion for the Tactile vibrations. Indeed, the Heel Strike alone simulation is preferred to the simulation of Heel Strike and Toe Strike. The metaphor used to design the vibration pattern does not influence these results. Thus, both metaphor can be used.

Concerning the subjective questionnaire, we performed a Friedman test. The reported p-values were adjusted for multiple comparisons. We found a significant effect for 4 criteria: Global appreciation $\left(\chi^{2}=3.99, p<0.001\right)$, Presence $\left(\chi^{2}=4.2, p<0.001\right)$, Realism $\left(\chi^{2}=4.65, p<0.001\right)$ and Walking sensation $\left(\chi^{2}=4.83\right.$, $p<0.001)$. Post-hoc analysis showed that $K_{T}(R C M, 1)$ was preferred to $C t r l$ for global appreciation $(p=0.001)$, presence $(p<$ $0.001)$, realism $(p<0.001)$ and walking sensation $(p<0.001)$. $K_{T}(R C M, 1)$ was also significantly better rated than $K_{T}(G R F M, 2)$ for realism ( $p=0.009)$. Moreover, $K_{T}(R C M, 2)$ was significantly better rated than $C t r l$ for walking sensation $(p=0.01)$. Finally, $K_{T}(G R F M, 1)$ was preferred to $C t r l$ for global appreciation ( $p=$ $0.03)$, presence $(p<0.001)$, realism $(p<0.001)$ and walking sensation $(p<0.001) . K_{T}(R C M, 1)$ was also significantly better rated than $K_{T}(R C M, 2)$ for global appreciation $(p=0.03)$. The results of the questionnaires are displayed in Figure 10.

The subjective questionnaires did not reveal any clear significant preference on the number of contacts for any of the experiments. The results suggest a small preference for 1 contact over 2 contacts, only for the Tactile vibrations. 


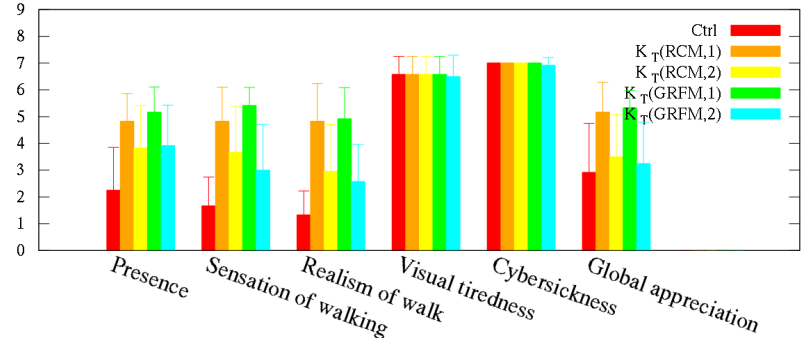

Figure 10: Results of the questionnaires for the third experiment. For each of the criteria, the mean and standard deviation for each condition are represented.

\subsubsection{Conclusion}

To sum up, the Tactile KKE provide a better sensation of walking when only 1 contact of the foot with the ground is simulated. More complex simulations are perceived as less natural for the users.

\subsection{Experiment 4: Testing Participant Preference for Multimodal KKE}

This last experiment focused on multimodal rendering of KKE. For this experiment, we introduced audio feedback of prerecorded footsteps to the KKE. The footstep sounds were synchronized with the other KKE by the Step Simulator. We used audio, visual and vibrotactile modalities individually or all together. For each modality, we selected the best components found in the previous experiments to yield the best sensation of walking in the VE and we tested every possible combinations of modalities.

Taken together, our previous results suggest guidelines for the best Visual and Tactile KKE. Heel strikes (1 contact) only should be used for the Tactile vibrations. Moreover, Vertical oscillations should be used for the Visual vibrations. Because the metaphor used has no clear significant effect on the Tactile vibrations, we chose to use the Rigid Contact Model for both modalities to simplify our model. Moreover, to keep the model as simple as possible, we chose to use only heels strikes for the Visual Vibrations also.

\subsubsection{Population}

Ten new participants ( 9 males and 1 female) aged from 21 to 27 (mean $=24.1$, standard deviation=2.2) performed the experiment. None of the participants had any known perception disorder. All participants were used to VEs but were naïve with respect to the proposed techniques, as well as to the experimental setup.

\subsubsection{Experimental Conditions}

We used a within subject design where the participants could freely navigate on a museum scene (Figure 11). They had the possibility to switch at will from one condition to the others. The visual $(V)$ modality was composed of $K_{V}(V, 1)$, the haptic (vibrotactile) $(H)$ modality was composed of $K_{T}(R C M, 1)$, and the audio $(A)$ modality was rendered using recorded playback of a wooden floor. All the possible combinations of these 3 modalities were available, from one modality alone to the 3 combined together, resulting in the following conditions: $V, H, A, V H, V A, H A$ and $V H A$. The experiment lasted approximatively 15 minutes.

\subsubsection{Collected Data}

The participants had to grade from 1 (very bad) to 7 (very good) the different conditions based on the following criteria: (1) Presence, (2) Sensation of walking, (3) Realism of the walk, (4) Fun and (5) Global appreciation.

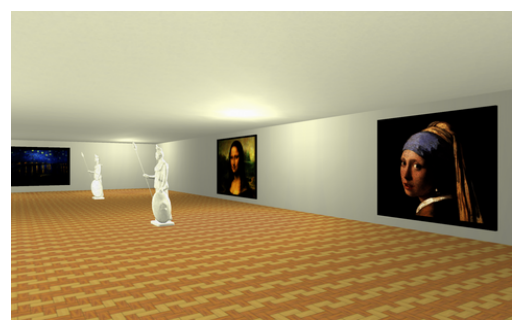

Figure 11: Museum scene of the 4th experiment.

\subsubsection{Results}

Concerning the subjective questionnaires, we performed a Friedman test. The reported $\mathrm{p}$-values were adjusted for multiple comparisons. We found a significant effect for all criteria: Fun $\left(\chi^{2}=4.49\right.$, $p<0.001)$, Global appreciation $\left(\chi^{2}=2.97, p=0.047\right)$, Presence $\left(\chi^{2}=4.90, p<0.001\right)$, Realism $\left(\chi^{2}=5.29, p<0.001\right)$ and Walking sensation $\left(\chi^{2}=4.90, p<0.001\right)$. Post-hoc analysis showed that $H$ was preferred to $V$ for fun $(p=0.02)$. $V A$ was preferred to $V$ for fun $(p=0.03)$, global appreciation $(p=0.047)$, and walking sensation $(p=0.04)$. $H A$ was preferred to $V$ for fun $(p<0.001)$, presence $(p=0.007)$, realism $(p<0.001)$, and walking sensation $(p=0.001) . H A$ was also significantly better rated than $V H$ for fun $(p=0.008)$. VHA was preferred to $V$ for presence $(p<0.001)$, realism $(p<0.001)$, and walking sensation $(p<0.001)$. VHA was significantly better rated than $H$ for presence $(p=0.01)$, realism $(p=0.03)$, and walking sensation $(p=0.04)$. VHA was significantly better rated than $A$ for presence $(p=0.03)$, realism $(p=0.01)$, and walking sensation $(p=0.009)$. VHA was significantly better rated than $V H$ for realism $(p=0.04)$ and walking sensation $(p=0.02)$. VHA was significantly better rated than $V A$ for realism $(p=0.002)$. VHA was significantly better rated than $H A$ for fun $(p=0.003)$.

The multimodal evaluation of the KKE showed that the the effects produced by each modality are reinforced when used in conjunction with the other modalities. In particular, conjunctions of two modalities scored higher on the fun criteria. Finally, the combination of the three modalities resulted in higher grades for presence, realism and walking sensation compared to each modalities taken alone or by two. Thus, our results suggest that a multimodal approach for the perception of the walk in the VE is preferred by the participants. The results of the questionnaires are displayed in Figure 12.

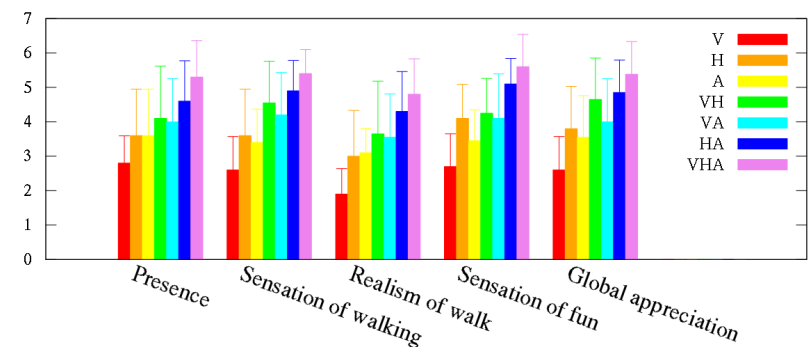

Figure 12: Results of the questionnaires for the fourth experiment. For each of the criteria, the mean and standard deviation for each condition are represented.

\subsubsection{Conclusion}

To sum up, the sensation of walking is increased when the different modalities of the KKE are taken together. A multimodal simulation is not only possible but is also recommended. 


\section{Discussion}

Our results suggest that the KKE allow more immersive and more enjoyable navigation compared to the classic FPS paradigm. Moreover, it clearly increases the sensation of actually walking inside the VE, while only using low cost devices. Finally, the KKE can be used to simulate different morphologies of virtual avatars, like height or weight, while still using the FPS paradigm.

The results of the experiments show that for the Visual KKE, the Vertical vibrations are always preferred to the Horizontal ones, and the type of contact of the feet with the ground does not change this result. Indeed, with Heel Strike only or with both Heel Strike and Toe Strike simulations, the Vertical is always preferred. The Vertical vibrations were designed to reproduce the point of view of a big creature walking like King Kong, while the Horizontal vibrations are more related to an external point of view. Thus, it seams that the Vertical vibrations are more immersive and thus provide a better sensation of walking in the VE.

Moreover, the standard Camera Motions do not change this result. Using the KKE in combination with standard Camera Motions does not modify the way a user perceives the KKE, and the most efficient type of KKE remains the same. This result gives a good hope that the KKE could be associated with many other techniques while keeping its properties. In particular, the best type of Visual KKE can probably be used safely in most situations.

Concerning the Tactile vibrations, the number of contacts was significant but the model used was not found to be significant. Thus, it seems that the simplicity of the model is a key to provide the best sensation of walking. However, some more complex models could be tested to simulate more complex virtual grounds, such as aggregate grounds made of sand or gravel for example [11]. The model of vibration could also be adapted dynamically to reproduce more accurately all the changes of the properties of the virtual ground during the navigation.

Interestingly, for some participants, the KKE modified their self perception inside the VE. Some participants quoted that they "felt like if they were heavier", or "like if they were a big creature like a troll". One of the women who participated also quoted that the double contact felt "as if walking with high heels".

Finally, our results clearly suggest the importance of a multimodal approach. In this paper we did not focus on the auditory modality. However, further integration of this modality would be highly interesting. Indeed, this modality fits particularly well into the scope of a desktop VR, and can provide a lot of useful information about the footsteps and the virtual ground properties.

\section{Conclusion ANd Future Work}

In this paper, we proposed a new navigation technique inspired from movies to increase the sensation of walking in a VE. This technique simulates each virtual step made in the VE by producing a visual and/or tactile vibration. The KKE simulates the contact with the ground of the heel and eventually the toe. Moreover, we proposed two different directions for the Visual vibrations corresponding to different points of view. Finally, we proposed two different models for the Tactile vibration patterns.

We conducted an evaluation of each of the components of the $\mathrm{KKE}$, as well as a multimodal evaluation in order to determine which components and parameters provide the best sensation of walking in VE. We found that vertical visual vibration simulating only heel contact were preferred by the participants. We also found that the Rigid Contact Model with only Heel Strike simulation was also preferred for the vibrotactile pattern. Finally, the participants showed that using the best patterns for each modality, multimodal feedback was preferred to navigations using only one modality.

Future work will focus on increasing the range of navigation motions such as running or jumping for example. Moreover, using the Step Simulator, a more developed auditory feedback rendering could be designed, or other parts of the body could be simulated.

Moreover, new models for the Tactile vibration patterns could be tested to check whether the vibrations could be improved to conveyed more information. In particular, different ground properties (like aggregate grounds for example) may be simulated with other different model, extending the perception of the virtual ground properties. Moreover, these models could be associated with real time audio synthesis to simulate the footstep sounds based on the ground properties.

\section{References}

[1] R. Alexander. Principles of Animal Locomotion. Princeton, 2003.

[2] D. A. Bowman, E. Kruijff, J. J. LaViola, and I. Poupyrev. 3D User Interfaces: Theory and Practice. Addison-Wesley Boston (MA), 2005.

[3] S. Hillaire, A. Lécuyer, T. R. Corte, R. Cozot, and G. Breton. A real-time visual attention model for predicting gaze point during firstperson exploration of virtual environments. In Proceedings of ACM Symposium on Virtual Reality Software and Technology (VRST), 2009.

[4] S. Hillaire, A. Lécuyer, R. Cozot, and G. Casiez. Using an eyetracking system to improve camera motions and depth-of-field blur effects in virtual environments. In Proceedings of the IEEE conference on Virtual Reality, pages 47-50, 2008.

[5] H. Iwata. Walking about virtual environments on an infinite floor. In Proceedings of the IEEE Virtual Reality, page 286, 1999.

[6] H. Iwata, H. Yano, H. Fukushima, and H. Noma. Circulafloor: A locomotion interface using circulation of movable tiles. In Proceedings of IEEE Virtual Reality, pages 223-230, 2005.

[7] H. Iwata, H. Yano, and F. Nakaizumi. Gait master: A versatile locomotion interface for uneven virtual terrain. In Proceedings of IEEE Virtual Reality, pages 131-137, 2001.

[8] A. D. Kuo. A simple model of bipedal walking predicts the preferred speed-step length relationship. Journal of biomechanical engineering, 123:264, 2001.

[9] A. Lécuyer, J.-M. Burkhardt, J.-M. Henaff, and S. Donikian. Camera motions improve the sensation of walking in virtual environments. In Proceedings of IEEE Virtual Reality, pages 11-18, 2006.

[10] M. Marchal, A. Lécuyer, G. Cirio, L. Bonnet, and M. Emily. Walking up and down in immersive virtual worlds: Novel interactive techniques based on visual feedback. In IEEE Symposium on $3 D$ User Interfaces (3DUI), pages 19-26, 2010.

[11] R. Nordahl, S. Serafin, and L. Turchet. Sound synthesis and evaluation of interactive footsteps for virtual reality applications. In Proceedings of IEEE Virtual Reality, pages 147-153, 2010.

[12] T. Novacheck. The biomechanics of running. Gait \& Posture, 7(1):7795, 1998.

[13] A. Okamura, J. Dennerlein, and R. Howe. Vibration feedback models for virtual environments. In Proceedings of the IEEE Conference on Robotics and Automation, volume 1, pages 674-679, 1998.

[14] S. Papetti, F. Fontana, M. Civolani, A. Berrezag, and V. Hayward. Audio-tactile display of ground properties using interactive shoes. Haptic and Audio Interaction Design, pages 117-128, 2010.

[15] S. Serafin, L. Turchet, and R. Nordahl. Extraction of ground reaction forces for real-time synthesis of walking sounds. In Proceedings of the 2009 Audio Mostly Conference, pages 99-105, 2009.

[16] S. Toshiaki and M. Tsutomu. The terrain surface simulator alf. In International Conference on Artificial Reality and Telexistence, volume 8, pages 170-174, 1998.

[17] C. Vaughan, B. Davis, and C. Jeremy. Dynamics of Human Gait. Human Kinetics Publishers Champaign, Illinois, 1992.

[18] Y. Visell, J. R. Cooperstock, B. L. Giordano, K. Franinovic, A. Law, S. McAdams, K. Jathal, and F. Fontana. A vibrotactile device for display of virtual ground materials in walking. Lecture Notes in Computer Science, 5024:420, 2008.

[19] Y. Visell, A. Law, J. Ip, S. Smith, and J. Cooperstock. Interaction capture in immersive virtual environments via an intelligent floor surface. In Proceedings of IEEE Virtual Reality, pages 313-314, 2010. 